\title{
Enhanced susceptibility of cancer cells to oncolytic rhabdo-virotherapy by expression of Nodamura virus protein B2 as a suppressor of RNA interference
}

\author{
Donald Bastin ${ }^{1,2+}$, Amelia S. Aitken ${ }^{1,2+}$, Adrian Pelin ${ }^{1,2}$, Larissa A. Pikor ${ }^{1,2}$, Mathieu J. F. Crupi ${ }^{1,2}$, Michael S. Huh ${ }^{1,2}$, \\ Marie-Claude Bourgeois-Daigneault ${ }^{1,2}$, John C. Bell ${ }^{1,2}$ and Carolina S. Ilkow ${ }^{1,2^{*}}$
}

\begin{abstract}
Antiviral responses are barriers that must be overcome for efficacy of oncolytic virotherapy. In mammalian cells, antiviral responses involve the interferon pathway, a protein-signaling cascade that alerts the immune system and limits virus propagation. Tumour-specific defects in interferon signaling enhance viral infection and responses to oncolytic virotherapy, but many human cancers are still refractory to oncolytic viruses. Given that invertebrates, fungi and plants rely on RNA interference pathways for antiviral protection, we investigated the potential involvement of this alternative antiviral mechanism in cancer cells. Here, we detected viral genome-derived small RNAs, indicative of RNAi-mediated antiviral responses, in human cancer cells. As viruses may encode suppressors of the RNA interference pathways, we engineered an oncolytic vesicular stomatitis virus variant to encode the Nodamura virus protein B2, a known inhibitor of RNAi-mediated immune responses. B2-expressing oncolytic virus showed enhanced viral replication and cytotoxicity, impaired viral genome cleavage and altered microRNA processing in cancer cells. Our data establish the improved therapeutic potential of our novel virus which targets the RNAi-mediated antiviral defense of cancer cells.
\end{abstract}

Keywords: Oncolytic virus, RNA interference, Vesicular stomatitis virus, B2

\section{Background}

Oncolytic viruses (OVs) possess an intrinsic or engineered ability to selectively target, replicate in and kill cancer cells [1]. These promising cancer therapeutics exploit cellular defects that promote tumour growth [2], destroy tumour-associated vasculature [3], induce anti-tumour immunity [1] and synergize with other treatments [4]. OVs must overcome barriers triggered by viral infection, including those mounted by cancer cells and components of the tumour microenvironment

\footnotetext{
* Correspondence: cilkow@uottawa.ca

${ }^{\dagger}$ Donald Bastin and Amelia S. Aitken contributed equally to this work.

${ }^{1}$ Centre for Innovative Cancer Research, Ottawa Hospital Research Institute, Ottawa K1H 8L6, Canada

${ }^{2}$ Department of Biochemistry, Microbiology and Immunology, University of

Ottawa, Ottawa K1H 8M5, Canada
}

[5]. The type I interferon (IFN) pathway is a well-characterized mammalian signaling cascade triggered upon viral attack to protect the surrounding cells and alert the immune system to contain infection [6]. The production of type I IFNs promotes an antiviral and anti-proliferative state in addition to inducing innate and adaptive immunity [2]. This antiviral response represents a major barrier to virus replication and spread in healthy tissues and is necessary to the safety of OV therapy [7]. Interestingly, the genetic alterations promoting tumourigenesis are associated with enhanced cancer cell susceptibility to viral infection [2]. Many pathways activated in response to infection that inhibit cell growth, activate apoptosis, and alert the immune system, are incompatible with malignant growth and are often defective in cancer cells [2]. Since these defects are common 
in cancer cells, they facilitate the targeted killing of cancer cells by certain OVs. Despite having IFN pathway defects, many cancers are still quite resistant to OV therapy [7]. For example, the vesicular stomatitis virus (VSV) is an OV platform with promising potential for clinical translation [8]. A VSV variant with an improved therapeutic index (VSV $\Delta 51$ ) is impaired in its capacity to block the IFN response and infect normal tissues [7]. The degree of susceptibility to VSV $\Delta 51$ killing varies among human cancers [7], due to the IFN status of the cancer cells and the potential involvement of other antiviral mechanisms within resistant tumours.

An alternative antiviral strategy relies on RNA interference (RNAi) [9], in order to combat infection in plants, fungi and invertebrates. This system is similar to the microRNA (miRNA) processing pathway used for post-transcriptional regulation in most eukaryotes. Viral double-stranded RNA generated during replication and transcription is bound and cleaved by the host cytoplasmic enzyme Dicer to form 22-23 nucleotides long RNA fragments [10]. These short RNA fragments are loaded into the RNA-induced silencing complex (RISC) where a single strand is selected to target homologous viral RNA and therefore prevent viral genome replication and translation [10]. To counteract this RNAi-mediated antiviral response, many plant and insect viruses have evolved viral suppressors of RNAi (VSRs) [11]. One such virus is the Nodamura virus, which primarily infects insects but is also highly virulent to certain mammals like suckling mice and hamsters [11-13]. Nodamura virus encodes a VSR known as B2, which binds double-stranded RNA and inhibits processing by Dicer which prevents the production of antiviral siRNAs [14-16].

RNAi- and protein-mediated immunity were long thought to be non-overlapping mechanisms, with insects and invertebrates using one strategy and mammals using the other. Interestingly, recent discoveries suggest that these mechanisms might not be mutually exclusive. In fact, antiviral RNAi has been shown to function in mammalian embryonic or undifferentiated cells [17]. Considering the discovery of mammalian antiviral RNAi in embryonic stem cells and the genetic similarities between cancer cells and embryonic stem cells [18-22]; we hypothesized a role for antiviral RNAi in cancer cells. To investigate antiviral RNAi and its effects on OV therapy, we engineered a recombinant VSV $\Delta 51$ to express the Nodamura virus B2 protein. Herein, we characterize this novel OV and demonstrate the interplay between the B2-expressing virus and RNA processing pathways in cancer. Our results show enhanced cancer-specific killing by our virus as well as improved viral replication in vivo. Together, our data strongly suggest the involvement of the RNAi pathway in the antiviral defense of cancer cells.

\section{Results \\ Evidence of a functional antiviral RNAi mechanism detected in VSV $\mathbf{5} 51$ infected cancer cells}

To investigate the potential involvement of antiviral RNAi mechanisms in cancer cells, we infected human cancer cell lines with a VSV variant impaired in its ability to block IFN response (VSVD51) and performed small-RNA deep sequencing. We showed that virus-derived small RNAs (vsRNAs) have a length bias towards 22-mers in several cell lines (Fig. 1a and Additional file 1: Figure S1A), consistent with the size of Dicer cleavage products. Importantly, this enrichment for 22-mers is present in positive strand vsRNAs, suggesting the occurrence of double stranded RNA cleavage. This is also characteristic of Dicer products, and likely acts during the synthesis of positive strands in VSV genome replication.

\section{B2 protein enhances VSV $\Delta 51$ replication in virus-resistant cancer cells}

We reasoned that if antiviral RNAi is triggered in mammalian cancer cells upon VSV $\Delta 51$ virus infection, expression of an RNAi viral suppressor should significantly enhance virus growth and cytotoxicity. To this end, we investigated the effects of the VSR protein B2 on VSV $\Delta 51$ replication in cancer cells, and characterized two human cancer cell lines (melanoma M14 cells and renal carcinoma 786-O cells) transfected with fluorescently-tagged B2 or empty vector (mock control). M14 and 786-O cell lines were selected as models for further characterization as they are both resistant to VSV infection and have functional type I IFN pathways. Upon drug-selection and sorting of positive cells, we confirmed ectopic expression of fluorescently-tagged constructs, as shown in fluorescence microscopy images (Additional file 1: Figure S1B). Viral outputs from B2-expressing M14 and 786-O cells infected with VSV $\Delta 51$ were significantly higher relative to mock controls (Fig. 1b), suggesting that B2 may enhance viral production.

\section{VSV $\Delta 51$-mediated expression of B2 enhances cytotoxicity} in cancer cell lines

As ectopic expression of the B2 protein enhanced VSV $\Delta 51$ titers in both M14 and 786-O cell lines, we engineered a VSV $\Delta 51$ virus variant encoding His-tagged B2 to assess effects of virus-mediated B2 expression. B2, or GFP as a control, was cloned between the G and L genes of the VSV $\Delta 51$ backbone (Fig. 1c), using a strategy previously shown to support expression of transgenes without impairing virus replication [7, 23]. We infected Vero cells with VSVA51-B2 and confirmed transgene expression by immunoblotting for His-tagged B2. As predicted, expression of B2 enhanced expression of VSV viral proteins (Fig. 1d). 


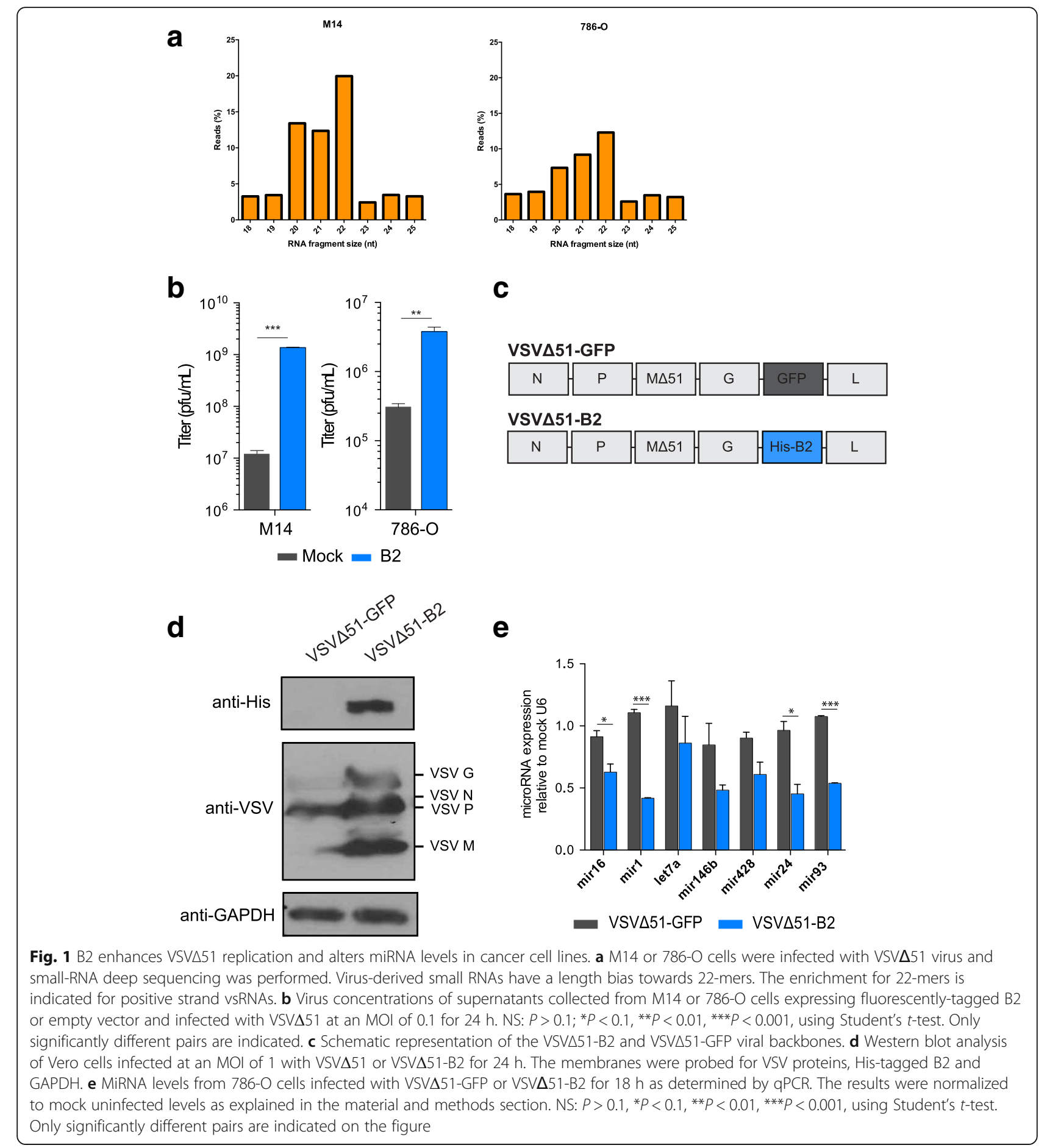

While the mechanism of action of VSVA51-encoded B2 on mammalian cancer cells remains to be elucidated, previous studies have shown that B2 blocks processing of small RNAs by Dicer [24, 25]. Given that B2 enhanced VSV $\Delta 51$ production by mammalian cancer cells, we investigated whether miRNA levels were affected by VSV $\triangle 51-\mathrm{B} 2$ using quantitative PCR (qPCR) for various miRNAs from infected 786-O cells. For the majority of miRNAs tested, including miR-1, miR-16, miR-24, and miR-93, the miRNA expression levels measured in VSVA51-B2 infected samples were significantly lower compared to VSV $\Delta 51-$ GFP samples (Fig. 1e), suggesting inhibition of small RNA processing by B2.

To determine if VSVA51-B2 could kill cancer cells more efficiently than VSV $\Delta 51-G F P$, we screened a panel of 38 different human cancer cell lines. The cells were 
infected at a multiplicity of infection (MOI) of 1 and cell viability was assessed. B2-expressing virus showed enhanced killing in the majority of cancer cell lines tested, including M14 and 786-O cells (Fig. 2a). In our screen, we also included an additional virus variant encoding vaccinia Copenhagen virus VP55, a different
VSR [26], which similarly enhanced virus-mediated cell killing in our studies (Fig. 2a).

To determine whether VSV $\Delta 51-\mathrm{B} 2$ could affect virus production, we assessed viral replication at multiple time points in M14 and 786-O cells, and found that VSVA51-B2 significantly enhanced replication over time,

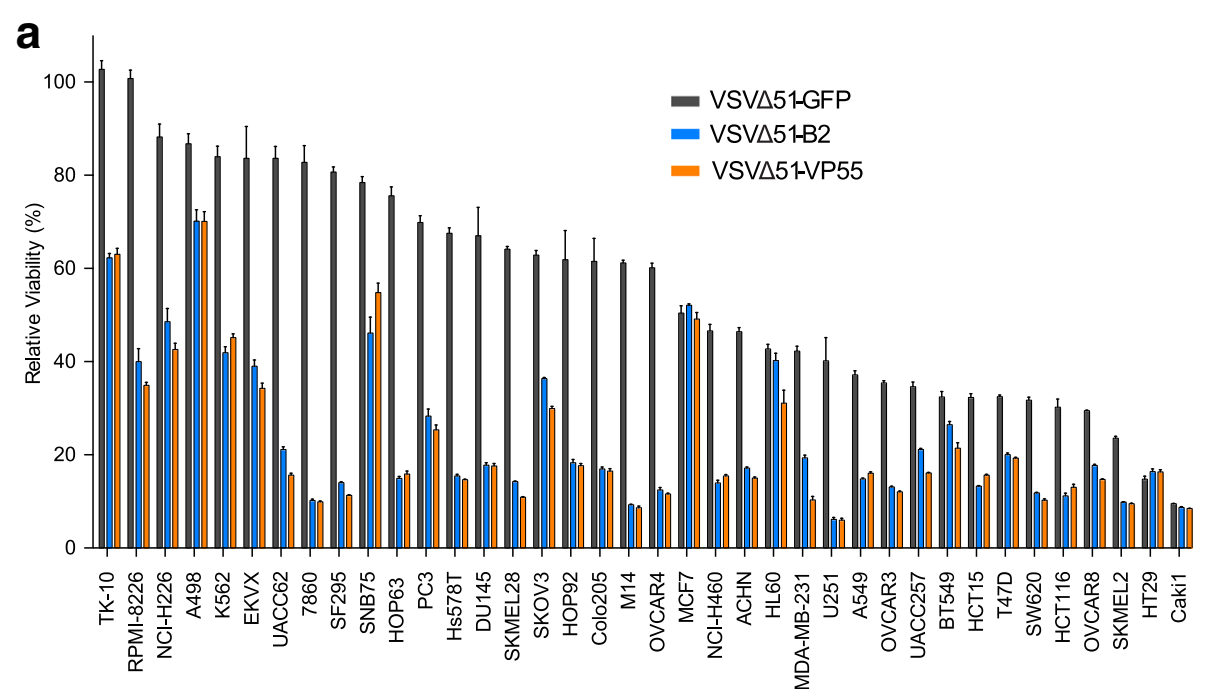

b
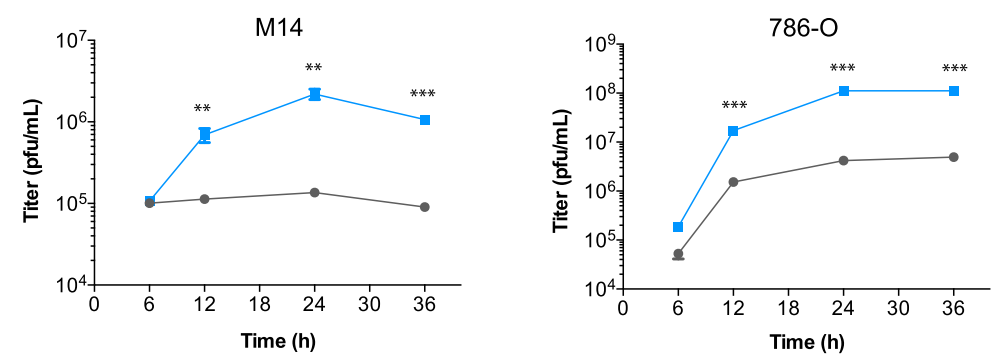

C

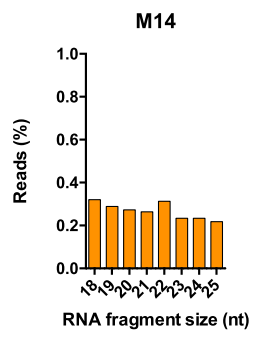

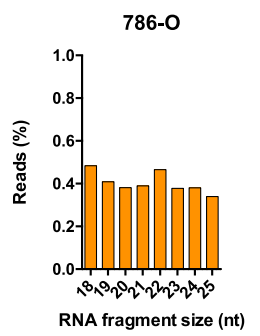

d

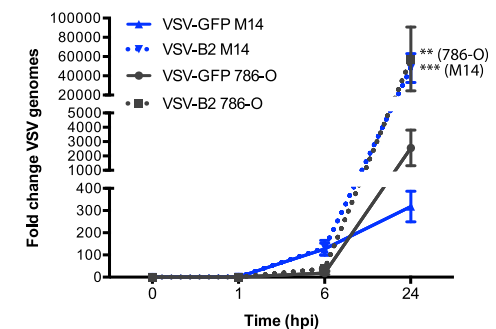

Fig. 2 VSV $\triangle 51-B 2$ alters cytotoxicity and viral genome cleavage. a Relative metabolic activity of 38 human cancer cell lines infected with VSV $551-$ GFP or VSVA51-B2 or additionally VSVA51-VP55 for $48 \mathrm{~h}$ at an $\mathrm{MOI}$ of 1 . The results are expressed as a percentage of the signal obtained compared to mock treatment. b Time-course of viral titers from 786-O and M14 cell lines infected with VSVA51-GFP or VSVA51-B2 at an MOI of 3. NS: $P>0.1,{ }^{*} P<0.1,{ }^{*} P<0.01,{ }^{* *} P<0.001$, using Student's $t$-test. Only significantly different pairs are indicated on the fig. c We performed small-RNA deep-sequencing using M14 or 786-O cells infected with VSVA51-B2 at an MOI of 0.1 for $18 \mathrm{~h}$. B2 expression in VSV 51 virus abrogates genomic cleavage as 22-mer vsRNAs are no longer prominent. VSV $\Delta 51-B 2$ derived vsRNAs display a bias towards positive strand reads in M14 and $786-O$ cells. $\mathbf{d}$ The indicated human cancer cell lines were infected with VSVD51-GFP or VSVD51-B2 (MOI=0.1). At the indicated time points, the expression level of virus genomes for each sample was quantified and normalized to GAPDH. Levels of VSV genomes are expressed relative to the level observed in the VSVD51-GFP 1 h-post-infection samples, which were arbitrarily set to 1 . Error bars indicate \pm SD among triplicates. NS: $P>0.1,{ }^{*} P<0.1,{ }^{* *} P<0.01,{ }^{* *} P<0.001$, using Student's $t$-test 
relative to VSV $\triangle 51-$ GFP (Fig. 2b). Further, we infected GM38 fibroblasts with VSVA51-B2 or VSV $551-$ GFP to investigate if $\mathrm{B} 2$ expression affects virus replication in healthy cells, and found that VSVA51-B2 infection did not significantly increase viral cytotoxicity at an MOI of 1 (Additional file 1: Figure S2A).

VSV 5 1-B2 prevents VSV genome cleavage in cancer cells To determine whether B2 protects VSV from genome cleavage, we performed small-RNA deep-sequencing on VSVA51-B2 infected cancer cells similar to the experiment in Fig. 1. We showed that B2 expression in VSV $\Delta 51$ virus abrogates genomic cleavage as 22-mer vsRNAs are no longer prominent in different cell lines (Fig. 2c and Additional file 1: Figure. S2B). Interestingly, VSV $\Delta 51-B 2$ derived vsRNAs display a bias towards positive strand reads compared to VSV 551 vsRNAs in cancer cells (Fig. 2c and Additional file 1: Figure S2B). Since the viral positive strand consists of viral mRNAs and positive sense genome copies, a larger bias for positive sense vRNAs suggests more efficient mRNA transcription in our VSV $\Delta 51-B 2$ virus. In addition, we infected M14 and 786-O cells with VSV $\Delta 51-G F P$ or VSV $\Delta 51-B 2$ and showed that the expression level of virus genomes for each sample was enhanced in response to VSV $\Delta 51-B 2$, relative to VSV $\Delta 51$-GFP (Fig. 2d). Taken together, these data suggest that VSVA51-B2 inhibits direct cleavage of the viral genome and host RNA processing pathways.

\section{VSV $\Delta 51-B 2$ and the type I IFN response}

To characterize the effect of B2 expression on mammalian cancer cells at a transcriptome level, we performed a microarray analysis on samples from M14 cells infected with either VSVA51-GFP or VSVA51-B2. Our results show that at a low MOI, VSV $\Delta 51-B 2$ virus induced the expression of a variety of immune related genes, which were not affected by VSV $\Delta 51$ infection (Fig. 3a). Through GO-term analysis, we detected an upregulation of genes by at least four-fold in response to VSV $\Delta 51-B 2$, but not VSVA51-GFP. We also showed enrichment of cytokine and cytokine activity predominantly associated with an IFN response (Fig. 3b). Interestingly, at high MOI, most immune genes upregulated by VSV $\Delta 51-\mathrm{B} 2$ at low MOI remain unchanged with few visible differences between the viruses (Fig. 3a).

Given that the IFN response is an important antiviral mechanism in mammalian cells, we investigated the potential impact of B2 on IFN responsiveness by qPCR after infection of 786-O cells. Consistent with our microarray results, we observed a significant increase in IFN- $\beta$ levels $24 \mathrm{~h}$ post-infection with VSV $\Delta 51-\mathrm{B} 2$ relative to the control virus (Fig. 3c). We also showed that VSV $\Delta 51-\mathrm{B} 2$ enhances IFN- $\beta$ secretion in $786-\mathrm{O}$ cells, relative to VSVA51-GFP, by ELISA (Fig. 3d). Lastly, we investigated if the production of VSV $\Delta 51-\mathrm{B} 2$ could be further enhanced by blocking the IFN pathway. We have previously shown that B19R, a soluble type I IFN scavenger expressed by vaccinia Copenhagen virus, enhances VSV $\Delta 51$ production [26]. In order to block the antiviral effect of the IFN that would be produced in response to VSV $\Delta 51$ infection, we generated conditioned-media from vaccinia virus-infected HeLa cells and pre-treated 786-O cells with B19R-containing media. We found that viral titers were significantly higher for VSV $\Delta 51-\mathrm{B} 2$ with both control media and vaccinia Copenhagen virus conditioned-media compared to VSVA51-GFP (Fig. 3e); however, the absolute increase in virus titers after exposing the cells to vaccinia Copenhagen virus-conditioned media was similar for both VSVA51-GFP and VSVA51-B2. Although induction of antiviral RNAi has been shown in mature mammalian cells $[27,28]$, our data suggest that the RNAi-based pathway is an IFN-independent antiviral mechanism in cancer cells as B2 expression alone enhances viral titers, which can be further enhanced by blocking the IFN response.

\section{Virus-mediated B2 expression enhances replication and cytokine production in vivo}

To establish an in vivo mouse model, we first screened RENCA mouse renal carcinoma cells in vitro to determine whether VSVA51-B2 enhanced cytotoxicity. We showed that, as observed using human cell lines, RENCA cells were more efficiently killed by the B2-expressing virus (Additional file 1: Figure S2C). In vivo, we subsequently tested the RENCA cell line which is syngeneic to Balb/c mice. In addition, we used the human M14 melanoma cell line as a xenograft model in nude mice. For both models, VSV $\Delta 51-B 2$ titers from subcutaneous tumours harvested $24 \mathrm{~h}$ post-intratumoural viral injection were significantly higher compared to VSVA51-GFP (Fig. 4a). Consistent with our microarray analysis, the IFN- $\gamma$, TNF- $\alpha$ and MCP-1 concentrations from the serum of the RENCA tumour-bearing Balb/c mice were significantly increased for the VSV $\Delta 51-\mathrm{B} 2$ treated mice compared to VSV $\triangle 51-G F P$ treated mice (Fig. 4b). In contrast, Il-6 concentrations did not significantly increase (Fig. 4b). Biodistribution analyses following intravenous administration revealed unchanged amounts of virus obtained from the different organs for VSV $\Delta 51$ and VSV $\Delta 51-B 2$ (Additional file 1: Figure S2D-E).

\section{Discussion}

In this study, we demonstrate that B2 expression is sufficient to enhance VSV $\Delta 51$ replication and cytotoxicity (Fig. 1-2) in mammalian cancer cells. Our screen of human cancer cells demonstrated enhanced efficacy of VSV $\Delta 51-B 2$ in killing the majority of cancer cell lines tested. The enhanced cytotoxic ability of VSV $\Delta 51-\mathrm{B} 2$ 

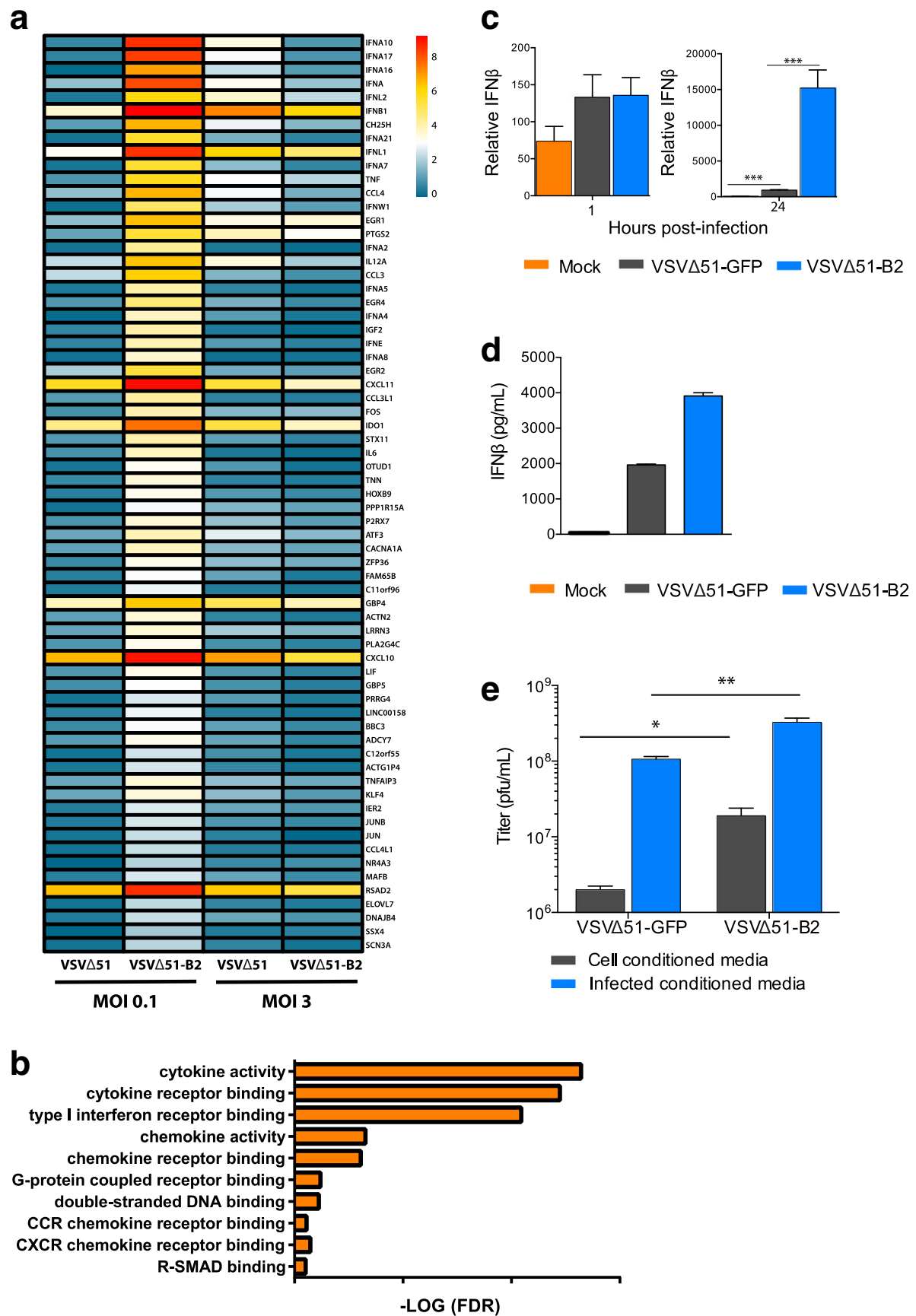

Fig. 3 VSVA51-B2 modulates IFN response and cytokine production. a Microarray analysis of M14 cells infected with VSVA51-GFP or VSVA51-B2 at low and high $\mathrm{MOI}$ as indicated. $\mathbf{b}$ Enrichment of cytokine and cytokine activity in the microarray, associated with an IFN response. $\mathbf{c}$ qPCR analysis of IFN- $\beta$ expression of 786-O cells infected for various times. IFN- $\beta$ levels were normalized to GAPDH levels within each sample. $\mathbf{d}$ ELISA for IFN- $\beta$ from supernatants of 786-O cells infected with VSV $\Delta 51-$ GFP or VSVA51-B2 at an MOI of 0.1 for $24 \mathrm{~h}$. e Virus outputs of VSV $\Delta 51-$-GFP and VSV $551-$ B2 obtained from 786-O cells pre-treated with vaccinia Copenhagen virus conditioned-media. Virus-cleared supernatants from HeLa cells that were infected with vaccinia Copenhagen virus at an $\mathrm{MOI}$ of $1 \mathrm{for} 48 \mathrm{~h}$ or left uninfected were transferred onto 786-O cells prior to infection with VSV $\Delta 51$-GFP or VSV $\Delta 51-B 2$ for 48 h. NS: $P>0.1,{ }^{*} P<0.1,{ }^{* *} P<0.01,{ }^{* * *} P<0.001$, using Student's $t$-test. Only significantly different pairs are indicated on the figure

suggests that RNAi may be an important factor hindering virus replication in resistant cancer cells. It is important to note that the cell lines in which there was no difference in cytotoxicity were the most sensitive to viral infection (Fig. 2), suggesting that the lack of improvement could potentially be attributed to the already 

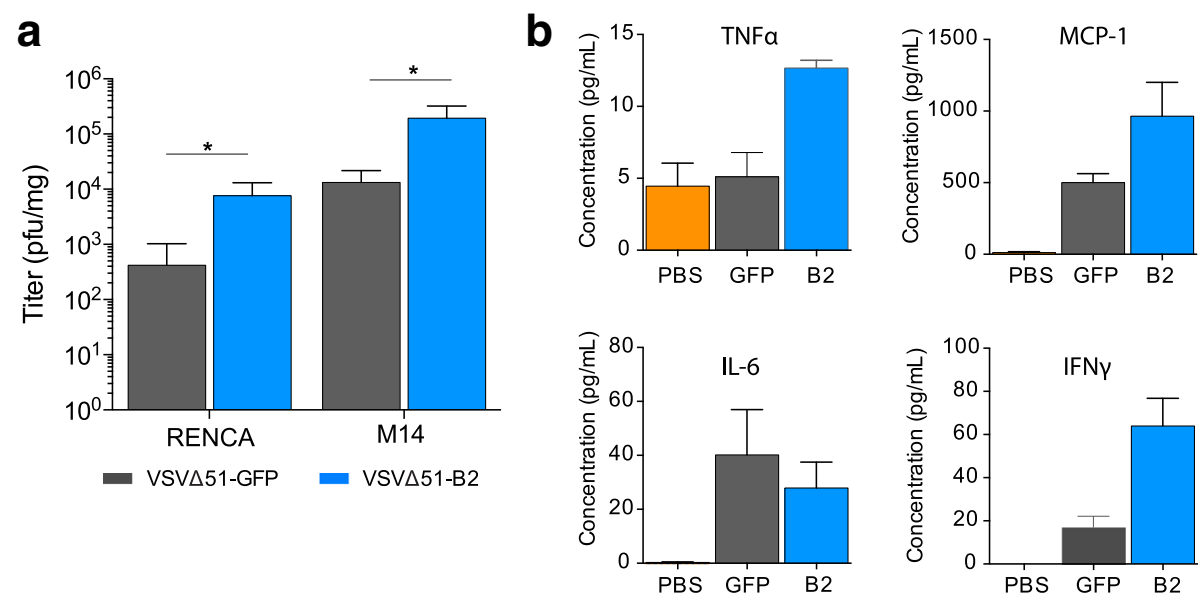

Fig. 4 VSV $\triangle 51-B 2$ enhances replication and cytokine levels within in vivo tumour models. a Viral titers obtained 24 hpi from subcutaneous M14 or RENCA tumours. Virus was administered intratumourally at a dose of 1E9 pfu of VSV $\Delta 51-$ GFP or VSV $\Delta 51-B 2$. NS: $P>0.1,{ }^{*} P<0.1,{ }^{* *} P<0.01$, ${ }^{*} * P<0.001$, using Student's $t$-test. Only significantly different pairs are indicated on the fig. $\mathbf{b}$ Serum levels of TNF-a, MCP-1, IL-6 and IFN- $\gamma$ from RENCA tumour-bearing C57BL/6 mice. Virus was administered intratumourally at a dose of 1E9 pfu of VSVA51-GFP or VSV $\Delta 51-B 2$ and serum collected 24 hpi. NS: $P>0.1,{ }^{*} P<0.1,{ }^{* *} P<0.01,{ }^{* *} P<0.001$, using Student's $t$-test. Only significantly different pairs are indicated on the figure

maximal virus production by these cells. An antiviral RNAi system may still function in these cells but may only be apparent in certain conditions, such as within the tumour microenvironment where many factors come together and create additional barriers to infection.

Interestingly, virus variants encoding B2 and VP55, two VSRs that impair the RNAi response by different mechanisms, show the same improvement in killing ability for all cell lines tested (Fig. 2). The mechanism of B2 involves binding of small RNA fragments which could either prevent their processing by Dicer or loading into RISC. On the other hand, VP55 polyadenylates miRNAs which targets them for degradation [29]. Given that both VSRs improve VSVA51-mediated killing to the same extent, this suggests that inhibition of the RNAi pathway improves virus replication regardless of the mechanism through which inhibition is achieved. VP55 does not polyadenylate all small RNAs and key features such as the presence of a 2'O methyl group protect a subset of small RNAs from degradation [29]. Notably, vsRNAs have been previously shown to be 2'O methylated, which protects them from degradation [30]. This may be advantageous for cell lines in which direct viral genome cleavage occurs as ideally, cleaved genome fragments can provide additional protection through targeting homologous viral genomes and transcripts.

To begin exploring the mechanism of B2 on VSVA51 replication, we investigated the potential impact of B2 on the IFN response. A number of cellular proteins such as Toll-like receptor 3, retinoic acid inducible gene I, 2 '-5' oligoadenylate synthetase and protein kinase $R$ recognize dsRNA and trigger a potent antiviral immune response [31]. Therefore, B2 may sequester the dsRNA substrates of these antiviral factors or interact with these proteins to prevent the sensing of dsRNA. As such, we investigated the effects of $\mathrm{B} 2$ on the IFN response, which is downstream of these pathways. We demonstrate that B2 does not suppress but actually significantly increases IFN- $\beta$ production (Fig. 3) compared to control virus, which is likely a result of enhanced replication. More specifically, at low MOI we detect an upregulation of immune genes with VSV $\Delta 51-B 2$ due to the ability of the VSV $\Delta 51-B 2$ virus to replicate in M14 cells and establish a successful infection which triggers a more robust IFN response (Fig. 3). However, at high MOI we do not see upregulation of immune genes by either VSV $\Delta 51-\mathrm{B} 2$ or VSV $\Delta 51-$ GFP virus (Fig. 3). Higher MOI is often used to overcome resistance to infectivity and often leads to faster cell death, suggesting there was insufficient time to mount a type I IFN response as most, if not all, cells were infected in the first round of replication. Of note, low MOI levels are more comparable to in vivo systems, in which VSV $\triangle 51-B 2$ virus may be more immunogenic. This prediction is in line with higher cytokine (IFN- $\gamma$, TNF- $\alpha$ and MCP-1) levels found in the serum of tumour bearing VSV $\Delta 51-\mathrm{B} 2$ treated immune-competent mice (Fig. 4).

Additionally, our vaccinia Copenhagen virus-conditioned media transfer experiments demonstrated that the blocking of type I IFN did not further enhance VSVA51-B2 replication compared to VSVA51-GFP. This is an indirect way to neutralize IFN-1 [26] and further suggested that IFN and B2 have different mechanisms of action (Fig. 3). Consistent with this notion, a similar induction of IFN-stimulated genes was observed in wildtype and RNAi-defective mouse embryonic fibroblasts [27]. In 
addition, suppression of RNAi by Nodamura virus protein B2 protein does not change the expression levels of IFN-stimulated genes in infected mice [17]. Importantly, our data do not eliminate the possibility of IFN-stimulated miRNAs limiting VSV $\Delta 51$ efficacy. Despite stimulating a type I IFN response, VSVD51-B2 has enhanced replication, suggesting that B2 expression is sufficient to overcome the antiviral response it stimulates.

One study has shown production of abundant influenza viral siRNAs in IFN-competent A549 cancer cells [27], but the existence of antiviral RNAi in cancer cells remains largely unexplored. We were able to detect viral genome fragments (vsRNAs) following infection with VSV $\triangle 51$ in a number of cell lines (Fig. 1 and Additional file 1: Figure S1), suggesting that viral genome cleavage is occurring. This may be facilitated by RNAi machinery being repurposed during viral infection. Interestingly, VSV $\Delta 51-\mathrm{B} 2$ infection resulted in a decrease in the percentage of vsRNAs (Fig. 2 and Additional file 1: Figure S2), suggesting the efficient prevention of this antiviral mechanism. A number of recent reports support the notion of antiviral genome cleavage in mammalian cells. It has been demonstrated that infection with RNA viruses can trigger the production of viral siRNAs, presumably as a result of direct virus genome cleavage $[17,32]$. The fact that many mammalian viruses encode VSRs, further supports the concept of a mammalian RNAi system. For example, Influenza A encodes the NS1 protein [33, 34], Ebola encodes VP35 [35, 36], HIV-1 encodes Tat [37, 38] and vaccinia Copenhagen virus encodes VP55 [29]. These proteins all have VSR-like functions, which suggests an evolutionary advantage of blocking antiviral RNAi.

It is possible that both direct inhibition of viral genome cleavage and inhibition of cellular antiviral miRNA production are co-occurring within certain cancer cell lines, as we observe changes in both VSV-specific and total RNA read length distributions (Additional file 1: Figure S1 and S2 and data not shown). The potential interplay between viruses and infected host cell miRNAs is a concept that is supported by several studies. For example, miR-29 has been reported to bind the 3' UTR of HIV mRNA which inhibits its translation and results in sequestering the mRNA into processing bodies [39]. IFN- $ß$ itself induces transcription of a number of miRNAs in hepatocytes that are complimentary to the hepatitis $C$ virus genomic RNA and inhibit viral replication [40]. In fact, activation of the IFN pathway has been shown to lead to the upregulation of a number of miRNAs including miR-1 [40-42], miR-129 [43], miR-146 [42] and miR-155 [42, 44, 45], some of which likely function to control infection. Perhaps most relevant to our study, 2 miRNAs (miR-24 and miR-93) have been previously shown to directly target the VSV genome and limit VSV replication [46]. Our results show that VSV $\Delta 51-B 2$ infection leads to the downregulation of both of these miRNAs, providing a potential explanation for the increased virus production using the $\mathrm{B} 2$ virus.

Overall, we demonstrate a novel role of the RNAi pathway as an intrinsic antiviral mechanism in cancer cells and how RNAi inhibition may be used to improve OV replication. Mechanistically, inhibition of direct viral genome cleavage and/or modulation of miRNA processing contribute to enhancing VSV $\Delta 51$ infection in a cell line specific manner. This work provides insight into the basic biology of viral defense mechanisms in cancer and promises to improve current $\mathrm{OV}$ therapies by tailoring viruses to overcome alternative antiviral mechanisms.

\section{Methods}

\section{Cell lines and culture}

All cell lines were purchased from the American Type Culture Collections (Manassas, VA). Mammalian cells were cultured in Dulbecco's modified Eagle's medium (DMEM) (Corning cellgro, Manassas, VA) or RPMI-1640 (K562, OVCAR3, OVCAR4, OVCAR8) (Corning cellgro, Manassas, VA) supplemented with $10 \%$ fetal bovine serum (FBS) (Sigma life science, St-Louis, MO) and maintained at $37^{\circ} \mathrm{C}$ with $5 \% \mathrm{CO}_{2}$. Drosophila melanogaster Schneider line 2 (S2) cells were cultured in SF900II serum-free medium (Invitrogen) at $25{ }^{\circ} \mathrm{C}$ under atmospheric pressures.

\section{DNA constructs and viral constructs}

The pcDNA3.1-puro B2 (Nodamura gene) plasmid used for generating the B2-expressing stable cell lines was made available by the Christopher Sullivan lab (Addgene plasmid \# 17228). The pEGFP-N1 plasmid (Catalog \# 6085-1) was purchased from Clontech (Moutain View, CA).

The B2 gene was amplified by PCR of the Nodamura virus genome. The primers were designed to include the XhoI and NheI restriction sites as well as to insert a $6 x$ histidine tag at the $5^{\prime}$ end of the B2 sequence. The digested PCR fragment was cloned into the XhoI and NheI digested VSV $\Delta 51$ backbone, as previously described. The primer pairs for inserting B2 into the VSV backbone are listed in Additional file 2: Table S1.

VP55 was PCR amplified from the Copenhagen strain of vaccinia virus and subcloned into pcDNA3.1 with an N-terminal Flag epitope. Flag-VP55 was subsequently PCR amplified and cloned into VSV $\triangle 51 \mathrm{M}$ using the same strategy.

\section{Transfection and selection of cell lines}

M14 and 786-O cells were transfected using Lipofectamine 2000 (Invitrogen, Carlsbad, CA) according to manufacturer instructions. Briefly, cells were plated in 6 well format 1 day prior to transfection. Plasmid and Lipofectamine reagent were incubated for $20 \mathrm{~min}$ and 
then added to plated cells in OptiMEM (Thermoscientific, Waltham, MA). $24 \mathrm{~h}$ post-transfection, medium was replaced by DMEM with 10\% FBS and cultured for $48 \mathrm{~h}$. Cells were then subjected to drug selection by the addition of Geneticin $(800 \mu \mathrm{g} / \mathrm{mL})$ (Thermo Fisher, Carlsbad, CA). Cells were expanded and GFP- or YFP-positive cells were sorted twice by FACS (MoFlo Astrios).

\section{Virus quantification}

Viral titers were obtained by plaque assays. Serial dilutions of the samples were prepared in serum-free DMEM. The dilutions were then transferred to monolayers of Vero cells and incubated at $37{ }^{\circ} \mathrm{C}$ for $1 \mathrm{~h}$. After the incubation, cells were overlaid with $0.5 \%$ agarose in DMEM supplemented with $10 \%$ FBS. Plates were incubated for $24 \mathrm{~h}$ at $37{ }^{\circ} \mathrm{C}$ with $5 \% \mathrm{CO}_{2}$ and plaques were counted.

\section{Virus rescue and purification}

Virus rescues were performed as previously described. Vero cells were infected with T7 polymerase-expressing vaccinia Copenhagen virus at an MOI of 3. Following a $2 \mathrm{~h}$ incubation, media was removed and cells were transfected with T7-driven plasmids encoding VSV N, P, and L genes as well as the VSV $\Delta 51-B 2$ backbone. Supernatants collected $48 \mathrm{~h}$ post-transfection were passed through a $0.22 \mu \mathrm{m}$ filter (MillexGP, Carrigtwohill, Ireland) to remove vaccinia Copenhagen virus.

For expansion and purification of the viral preparations, Vero cells were infected at an MOI of 0.001 and culture supernatants were collected $24 \mathrm{~h}$ post-infection. Supernatants were then filtered through a $0.2 \mu \mathrm{m}$ bottle top filter (Millipore, Etobicoke, Canada) and centrifuged at 30,100 g for $90 \mathrm{~min}$. The supernatant was discarded and pelleted virus was resuspended in Dulbecco's phosphate-buffered saline (Corning cellgro, Manassas, VA). Purified virus was kept at $-80^{\circ} \mathrm{C}$.

\section{Deep sequencing of vsRNAs}

Total RNA was extracted with TRIzol reagent (Invitrogen) according to manufacturer instructions. Library preparation for Illumina sequencing was performed (TCAG DNA Sequencing Facility, Toronto, ON). Briefly, RNA was enriched for 15-25 nt sizes before strand-specific, small-RNA library preparation and 50 bp single end read sequencing. Adapter trimming was done with Trimmomatic [47] following default parameters. Before read mapping, VSV $\Delta 51$ genome was constructed from the VSV reference genome (NC_001560), manually edited to delete the 51st methionine amino acid in the $M$ gene. Reads were mapped to VSV $\Delta 51$ genome using bbmap.sh script from the BBMap toolkit (http:// sourceforge.net/projects/bbmap) with a minimum alignment identity of $100 \%$. SAMtools was used to separate positive sense mapping from bbmap produced sam files
[48]. Lastly, positive sense reads were analyzed for size distribution using the reformat.sh script from the BBMap toolkit.

\section{Western blotting}

Cell pellets were lysed on ice for 30 min using complete protease inhibitor cocktail (Roche, Mississauga, Ontario, Canada) supplemented lysis buffer (1\% NP40, $150 \mathrm{mM}$ $\mathrm{NaCl}, 5 \mathrm{mM}$ EDTA, $50 \mathrm{mM}$ Tris $\mathrm{pH}$ 7.4). Lysates were centrifuged for $10 \mathrm{~min}$ at $16,000 \mathrm{~g}$ and cleared supernatants were mixed with dithiothreitol-supplemented loading buffer (250 mM Tris- $\mathrm{HCl} \mathrm{pH}$ 6.8, 10\% SDS, 30\% glycerol, 5\% $\beta$-Mercaptoethanol, $0.02 \%$ bromophenol blue). The samples were migrated on Bio-Rad Mini Protean 4-15\% TGX Protein Gels (Bio-Rad, Mississauga, ON) and transferred onto PVDF membranes (GE Healthcare, Buckinghanshire, UK) prior to blocking with 5\% skim milk powder (Oxoid Ltd., Basingstoke, UK) in Tris-buffered saline (TBS)with $0.1 \%$ Tween-20. The membranes were proofed using specific rabbit antibodies for $6 \times$ His tag (Abcam, Cambridge, UK), VSV (polyclonal anti-VSV serum for hyperimmune rabbits) [49]. Rabbit anti-GAPDH (Abcam, Cambridge, UK) and rat anti-tubulin (Novus Biologicals, Oakville, ON) antibodies were used as loading controls. Membranes were then probed with a horse radish peroxidase-coupled goat anti-rabbit secondary antibody (Millipore, Etobicoke, Canada) or goat anti-rat secondary antibody (Life Technologies, Carlsbad, CA) and the signal was revealed using Amersham ECL Western Blotting Detection Reagent (GE Healthcare, Buckinghamshire, UK). The gels were analyzed using FluorChem FC2 (Alpha Innotech, San Leandro, CA).

\section{Cell viability assay}

Relative metabolic activity of cells was used as a readout of cell viability and was determined using alamarBlue reagent (Bio-Rad, Mississauga, Ontario, Canada). The assays were performed according to manufacturer instructions. Briefly, cells were plated in 96-well plates and infected with the different viruses $24 \mathrm{~h}$ later. $48 \mathrm{~h}$ after virus infection, alamarBlue was added to each well to a final concentration of 1:10. The samples were incubated 1 to $5 \mathrm{~h}$ and the fluorescence readings (excitation and emission wavelengths of $530 \mathrm{~nm}$ and $590 \mathrm{~nm}$, respectively) were taken using a Fluoroskan Ascent FL (Thermo Labsystems, Beverly, MA).

\section{Quantitative PCR}

For miRNA qPCRs, RNA was extracted from infected cell pellets using TRIzol reagent (Life Technologies, Carlsbad, CA) according to the manufacturer instructions. The RNA concentration and purity was assessed using a NanoDrop ND-1000 spectrophotometer (Thermoscientific, Waltham, MA) prior to reverse transcription using Quanta miRNA cDNA synthesis kit (Gaithersburg, MD). 
For all other qPCRs, RNA was extracted using RNAeasy RNA extraction kit (QIAGEN, Toronto, ON, Canada) according to the manufacturer's instructions. The RNA concentration and purity was assessed using a NanoDrop ND-1000 spectrophotometer (Thermoscientific, Waltham, MA) prior to reverse transcription using RevertAid $\mathrm{H}$ Minus First Strand cDNA Synthesis kit (Thermoscientific, Waltham, MA).

qRT -PCR was performed on the non-pooled triplicate samples. Following conversion to cDNA by Superscript RT II (Invitrogen, Carlsbad, CA), qRT-PCR was carried out using Sybergreen (Invitrogen) according to the manufacturer's instructions. Analyses were performed on a Rotor-Gene RG-3000A machine (Corbett Research, Mortlake, AU) according to the manufacture instructions. The primer pairs specific for various gene products used in our experiments are listed in Additional file 2: Table S1. qRT-PCR measurements were normalized to the $U 6$ or GAPDH house-keeping genes for miRNA or RNA transcripts, respectively, using the Pfaffl method [50].

\section{Microarray}

Monolayers of M14 cells were treated at an MOI of 0.1 or 3 for $24 \mathrm{~h}$ with either VSV $\Delta 51$ or VSV $\Delta 51$ encoding B2 gene virus Total RNA was extracted with TRIzol reagent (Invitrogen) according to the manufacturer instructions. Experimental triplicate total RNA samples were processed by The Center for Applied Genomics at The Hospital for Sick Children for microarray analysis on a Human Prime View chip. Raw files were analyzed using the Transcriptome Analysis Console v3.0 (Affymetrix) software. Normalized transcript expression values further processed with $\mathrm{R}$. Heatmaps were produced using the R package "pheatmap" v1.0.8. GO Term Enrichment analysis (http://CRAN. R-project.org/package=pheatmap) was performed using the online EnrichR tool [51]. Genes selected for enrichment analysis are the subset of genes upregulated by the expression of the B2 gene in VSVA51 by at least 4-fold.

\section{ELISA}

The concentration of IFN- $\beta$ was determined using the human IFN- $\beta$ ELISA kit (R\&D Systems, Minneapolis, $\mathrm{MN}$ ) according to the manufacturer's instructions.

\section{Supernatant transfer experiments}

Vaccinia stocks were propagated in U-2 OS cells and cell-associated virus was collected by repeat [3] freezethaw cycles. Purification of viral stocks was done by centrifugation at $20,700 \mathrm{~g}$ through a $36 \%$ sucrose cushion (in $1 \mathrm{mM}$ Tris) before resuspension in $1 \mathrm{mM}$ Tris, $\mathrm{pH} 9$.

To generate infected cell conditioned media, U-2 OS cells were either mock treated or infected with VVdd-mCherry at a multiplicity of $10 \mathrm{PFU} /$ cell for $24 \mathrm{~h}$, harvested and then pelleted by centrifugation. Supernatants were collected and passed through a $0.22 \mu \mathrm{m}$ filter to eliminate cell-free vaccinia virions. To test for factors enhancing VSV infectivity, tumour cell monolayers were pre-treated for $2 \mathrm{~h}$ with conditioned U-2 OS supernatant. Tumour cells were then infected with VSV in the presence of conditioned medium.

\section{In vivo experiments and tumour models}

6-8 weeks old female Balb/c or nude mice (Charles River Laboratories, Wilmington, MA) were used. For the Balb/c mice $5 \times 10^{5}$ RENCA tumour cells were implanted subcutaneously 21 days prior to treatment. For the nude mice $1 \times 10^{8}$ M14 tumour cells were implanted subcutaneously 14 days prior to treatment. A single intratumoural injection of 1E8 PFU of VSVA51-GFP or VSVA51-B2 was performed. Tumours were harvested $24 \mathrm{~h}$ post-treatment, weighed and homogenized in PBS using a Powergen 125 tissue homogenizer (Fischer Scientific, Hampton, New Hampshire). Serial dilutions of tumour homogenates were tittered to obtain intratumoural titer data. For biodistribution experiments, naïve Balb/c mice were treated with a single intravenous injection of 1E8 PFU of VSVA51-GFP or VSVA51-B2. Mice were sacrificed $24 \mathrm{~h}$ or $48 \mathrm{~h}$ post-treatment and organs were harvested, frozen and homogenized in PBS as above. All experiments were approved by the University of Ottawa animal care and veterinary services (ME-2258).

Serum was obtained upon centrifugation of blood samples collected using lithium-heparin coated capillary tubes (Sarstedt, Newton) at $16000 \mathrm{~g}$ for $5 \mathrm{~min}$. The concentrations of IFN $\gamma$, TNF $\alpha, \mathrm{MCP}-1$ and IL- 6 were measured using a mouse inflammation cytometric bead array kit (BD Biosciences, San Jose, CA) according to manufacturer instructions. The results were acquired on a LSR Fortessa flow cytometer and analyzed using the FCAP array software (BD Biosciences).

\section{Additional files}

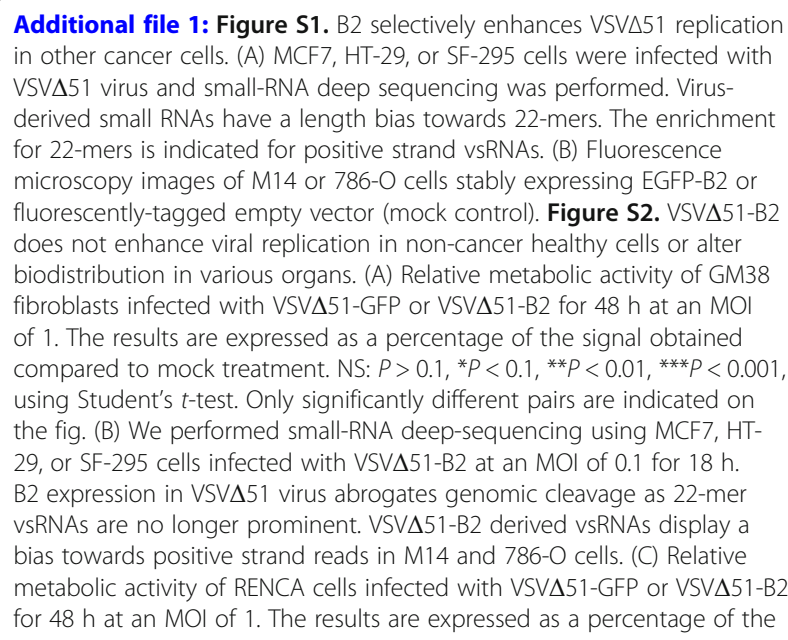


signal obtained compared to mock treatment. (D\&E) Biodistribution of VSV $\triangle 51-B 2$ in tumour-bearing C57BL/6 mice. Viral titers obtained from organs of tumour-bearing C57BL/6 mice, D] 24 or E] 48 hpi. Virus was administered intravenously at a dose of 1 E9 pfu of VSV $\Delta 51-$ GFP or VSVA51-B2. For organs where virus was undetectable, the titer was considered to be the value of the limit of detection of titering for this assay (5E1 pfu/organ). NS: $P>0.1,{ }^{*} P<0.1,{ }^{* *} P<0.01,{ }^{* * *} P<0.001$, using

Student's t-test. (PDF $5336 \mathrm{~kb}$ )

Additional file 2: Table S1. List of primers used in qRT-PCR assays. (DOCX $14 \mathrm{~kb})$

\section{Abbreviations}

IFN: Interferon; miRNA: MicroRNA; OV: Oncolytic virus; qPCR: Quantitative PCR; RISC: RNA-induced silencing complex; RNAi: RNA interference; VSR: Viral suppressors of RNAi; vsRNA: Virus-derived small RNAs; VSV: Vesicular stomatitis virus

\section{Acknowledgements}

We would like to thank the exceptional technical support of Catia Cemeus, Christiano Tanese de Souza and Julia Petryk, as well as members of the Bell, Auer, Atkins, Ilkow and Diallo laboratories for feedback on this project.

\section{Funding}

This work was funded by grants from the Terry Fox Research Foundation and the Canadian Institutes of Health Research to J.C.B. and C.S.I. J.C.B. is also supported by the Ontario Institute for Cancer Research and the Ottawa Regional Cancer Foundation. A.A. is funded by the Canadian Institutes of Health Research Frederick Banting and Charles Best Master's Award. A.P. is funded by the Joseph and Wolf Lebovic Cancer Genomics and Immunity Fellowship and Ontario Graduate Scholarship. L.P. and M.C.B.D. are funded by the Canadian Institutes of Health Research post-doctoral Fellowship.

\section{Availability of data and materials}

All data generated or analyzed during this study are included in this article and its Additional files 1 and 2 .

Deep-sequencing datasets of VSV genome reads generated during the current study are available in the Sequence Read Archive (SRA) repository (Accession number: SRP132124). Microarray data are deposited in the NCBI Gene Expression Omnibus (Accession number: GSE115138).

\section{Authors' contributions}

Conceptualization: DB, ASA, AP, LAP, MJFC, MSH, JCB, CSI; Methodology: DB, ASA, AP, LAP, MJFC, MSH, JCB, CSI; Validation: DB, ASA, AP, LAP, MJFC, MSH, JCB, CSI; Formal analysis: DB, ASA, AP, LAP, MJFC, MSH, JCB, CSI.; Investigation: DB, ASA, AP, LAP, MJFC, MSH, JCB, CSI; Resources: DB, MSH; Data curation: DB, ASA, AP, LAP, MCBD; Writing - review \& editing: ASA, AP, MJFC, CSI; Visualization: DB, ASA, AP, MJFC, CSI; Supervision: JCB, CSI; Project administration: CSI; Funding acquisition: JCB, CSI. All authors read and approved the final manuscript.

\section{Ethics approval}

All animal studies were approved by the institutional animal care committee of the University of Ottawa (Protocol ID: ME-2258) and carried out in accordance with guidelines of the National Institutes of Health and the Canadian Council on animal care.

\section{Competing interests}

The authors declare that they have no competing interests.

\section{Publisher's Note}

Springer Nature remains neutral with regard to jurisdictional claims in published maps and institutional affiliations.

\section{Received: 1 February 2018 Accepted: 25 May 2018 Published online: 19 June 2018}

\section{References}

1. Lichty BD, Breitbach CJ, Stojdl DF, Bell JC. Going viral with cancer immunotherapy. Nat Rev Cancer. 2014;14(8):559-67.
2. Ilkow CS, Swift SL, Bell JC, Diallo JS. From scourge to cure: tumour-selective viral pathogenesis as a new strategy against cancer. PLoS Pathog. 2014; 10(1):e1003836

3. Arulanandam R, Batenchuk C, Angarita FA, Ottolino-Perry K, Cousineau S, Mottashed A, Burgess E, Falls TJ, De Silva N, Tsang J, Howe GA, BourgeoisDaigneault MC, Conrad DP, Daneshmand M, Breitbach CJ, et al. VEGFmediated induction of PRD1-BF1/Blimp1 expression sensitizes tumor vasculature to oncolytic virus infection. Cancer Cell. 2015;28(2):210-24.

4. Bourgeois-Daigneault MC, Roy DG, Aitken AS, El Sayes N, Martin NT, Varette O, Falls T, St-Germain LE, Pelin A, Lichty BD, Stojdl DF, Ungerechts G, Diallo JS, Bell JC. Neoadjuvant oncolytic virotherapy before surgery sensitizes triple-negative breast cancer to immune checkpoint therapy. Sci Transl Med. 2018;10(422)

5. Ilkow CS, Marguerie M, Batenchuk C, Mayer J, Ben Neriah D, Cousineau S, Falls T, Jennings VA, Boileau M, Bellamy D, Bastin D, de Souza CT, Alkayyal A Zhang J, Le Boeuf F, et al. Reciprocal cellular cross-talk within the tumor microenvironment promotes oncolytic virus activity. Nat Med. 2015;21(5): 530-6.

6. Ivashkiv LB, Donlin LT. Regulation of type I interferon responses. Nat Rev Immunol. 2014;14(1):36-49.

7. Stojdl DF, Lichty BD, tenOever BR, Paterson JM, Power AT, Knowles S, Marius R, Reynard J, Poliquin L, Atkins H, Brown EG, Durbin RK, Durbin JE, Hiscott J, Bell JC. VSV strains with defects in their ability to shutdown innate immunity are potent systemic anti-cancer agents. Cancer Cell. 2003;4(4): 263-75.

8. Stojdl DF, Lichty B, Knowles S, Marius R, Atkins H, Sonenberg N, Bell JC. Exploiting tumor-specific defects in the interferon pathway with a previously unknown oncolytic virus. Nat Med. 2000;6(7):821-5.

9. Wilson RC, Doudna JA. Molecular mechanisms of RNA interference. Annu Rev Biophys. 2013;42:217-39.

10. Yan N, Chen ZJ. Intrinsic antiviral immunity. Nat Immunol. 2012;13(3):214-22.

11. Jiang L, Wei C, Li Y. Viral suppression of RNA silencing. Sci China Life Sci. 2012;55(2):109-18.

12. Bailey L, Newman JF, Porterfield JS. The multiplication of Nodamura virus in insect and mammalian cell cultures. J Gen Virol. 1975;26(1):15-20.

13. Bailey L, Scott HA. The pathogenicity of Nodamura virus for insects. Nature. 1973;241(5391):545.

14. Lu J, Getz G, Miska EA, Alvarez-Saavedra E, Lamb J, Peck D, Sweet-Cordero A, Ebert BL, Mak RH, Ferrando AA, Downing JR, Jacks T, Horvitz HR, Golub TR. MicroRNA expression profiles classify human cancers. Nature. 2005; 435(7043):834-8

15. Sullivan CS, Ganem D. A virus-encoded inhibitor that blocks RNA interference in mammalian cells. J Virol. 2005;79(12):7371-9.

16. Aliyari R, Wu Q, Li HW, Wang XH, Li F, Green LD, Han CS, Li WX, Ding SW. Mechanism of induction and suppression of antiviral immunity directed by virus-derived small RNAs in Drosophila. Cell Host Microbe. 2008;4(4):387-97.

17. Li Y, Lu J, Han Y, Fan X, Ding SW. RNA interference functions as an antiviral immunity mechanism in mammals. Science. 2013;342(6155):231-4.

18. Boumahdi S, Driessens G, Lapouge G, Rorive S, Nassar D, Le Mercier M, Delatte B, Caauwe A, Lenglez S, Nkusi E, Brohee S, Salmon I, Dubois C, del Marmol V, Fuks F, et al. SOX2 controls tumour initiation and cancer stemcell functions in squamous-cell carcinoma. Nature. 2014;511(7508):246-50.

19. Ben-Porath I, Thomson MW, Carey VJ, Ge R, Bell GW, Regev A, Weinberg RA. An embryonic stem cell-like gene expression signature in poorly differentiated aggressive human tumors. Nat Genet. 2008; 40(5):499-507.

20. Somervaille TC, Matheny CJ, Spencer GJ, Iwasaki M, Rinn JL, Witten DM, Chang HY, Shurtleff SA, Downing JR, Cleary ML. Hierarchical maintenance of MLL myeloid leukemia stem cells employs a transcriptional program shared with embryonic rather than adult stem cells. Cell Stem Cell. 2009;4(2):129-40.

21. Schoenhals M, Kassambara A, De Vos J, Hose D, Moreaux J, Klein B. Embryonic stem cell markers expression in cancers. Biochem Biophys Res Commun. 2009;383(2):157-62.

22. Mizuno H, Spike BT, Wahl GM, Levine AJ. Inactivation of p53 in breast cancers correlates with stem cell transcriptional signatures. Proc Natl Acad Sci U S A. 2010;107(52):22745-50.

23. Bourgeois-Daigneault MC, Roy DG, Falls T, Twumasi-Boateng K, St-Germain LE, Marguerie M, Garcia V, Selman M, Jennings VA, Pettigrew J, Amos S, Diallo JS, Nelson B, Bell JC. Oncolytic vesicular stomatitis virus expressing interferon-gamma has enhanced therapeutic activity. Mol Ther Oncolytics. 2016;3:16001. 
24. Chao JA, Lee JH, Chapados BR, Debler EW, Schneemann A, Williamson JR. Dual modes of RNA-silencing suppression by flock house virus protein B2. Nat Struct Mol Biol. 2005;12(11):952-7.

25. Johnson KL, Price BD, Eckerle LD, Ball LA. Nodamura virus nonstructural protein B2 can enhance viral RNA accumulation in both mammalian and insect cells. J Virol. 2004;78(12):6698-704.

26. Le Boeuf F, Diallo JS, McCart JA, Thorne S, Falls T, Stanford M, Kanji F, Auer R, Brown CW, Lichty BD, Parato K, Atkins H, Kirn D, Bell JC. Synergistic interaction between oncolytic viruses augments tumor killing. Mol Ther. 2010;18(5):888-95.

27. Li Y, Basavappa M, Lu J, Dong S, Cronkite DA, Prior JT, Reinecker HC, Hertzog P, Han Y, Li WX, Cheloufi S, Karginov FV, Ding SW, Jeffrey KL. Induction and suppression of antiviral RNA interference by influenza a virus in mammalian cells. Nat Microbiol. 2016;2:16250.

28. Qiu Y, Xu Y, Zhang Y, Zhou H, Deng YQ, Li XF, Miao M, Zhang Q, Zhong B, Hu $Y$, Zhang FC, Wu L, Qin CF, Zhou X. Human virus-derived small RNAs can confer antiviral immunity in mammals. Immunity. 2017;46(6):992-1004. e1005

29. Backes S, Shapiro JS, Sabin LR, Pham AM, Reyes I, Moss B, Cherry S, tenOever BR. Degradation of host microRNAs by poxvirus poly(a) polymerase reveals terminal RNA methylation as a protective antiviral mechanism. Cell Host Microbe. 2012;12(2):200-10.

30. Daffis S, Szretter KJ, Schriewer J, Li J, Youn S, Errett J, Lin TY, Schneller S, Zust R, Dong H, Thiel V, Sen GC, Fensterl V, Klimstra WB, Pierson TC, et al. 2'O methylation of the viral mRNA cap evades host restriction by IFIT family members. Nature. 2010;468(7322):452-6.

31. Gantier MP, Williams BR. The response of mammalian cells to doublestranded RNA. Cytokine Growth Factor Rev. 2007;18(5-6):363-71.

32. Maillard PV, Ciaudo C, Marchais A, Li Y, Jay F, Ding SW, Voinnet O. Antiviral RNA interference in mammalian cells. Science. 2013;342(6155):235-8.

33. Lu Y, Wambach M, Katze MG, Krug RM. Binding of the influenza virus NS1 protein to double-stranded RNA inhibits the activation of the protein kinase that phosphorylates the elF-2 translation initiation factor. Virology. 1995; 214(1):222-8.

34. Bucher $E$, Hemmes $H$, de Haan $P$, Goldbach $R$, Prins $M$. The influenza a virus NS1 protein binds small interfering RNAs and suppresses RNA silencing in plants. J Gen Virol. 2004;85(Pt 4):983-91.

35. Cardenas WB, Loo YM, Gale M Jr, Hartman AL, Kimberlin CR, MartinezSobrido L, Saphire EO, Basler CF. Ebola virus VP35 protein binds doublestranded RNA and inhibits alpha/beta interferon production induced by RIG-I signaling. J Virol. 2006:80(11):5168-78.

36. Haasnoot J, de Vries W, Geutjes EJ, Prins M, de Haan P, Berkhout B. The Ebola virus VP35 protein is a suppressor of RNA silencing. PLoS Pathog. 2007;3(6):e86

37. Bennasser $Y$, Le SY, Benkirane M, Jeang KT. Evidence that HIV-1 encodes an siRNA and a suppressor of RNA silencing. Immunity. 2005;22(5):607-19.

38. Bennasser $Y$, Jeang KT. HIV-1 tat interaction with dicer: requirement for RNA. Retrovirology. 2006;3:95.

39. Nathans R, Chu CY, Serquina AK, Lu CC, Cao H, Rana TM. Cellular microRNA and P bodies modulate host-HIV-1 interactions. Mol Cell. 2009;34(6):696-709.

40. Pedersen IM, Cheng G, Wieland S, Volinia S, Croce CM, Chisari FV, David M. Interferon modulation of cellular microRNAs as an antiviral mechanism. Nature. 2007:449(7164):919-22.

41. Scagnolari C, Zingariello P, Vecchiet J, Selvaggi C, Racciatti D, Taliani G, Riva E, Pizzigallo E, Antonelli G. Differential expression of interferon-induced microRNAs in patients with chronic hepatitis $C$ virus infection treated with pegylated interferon alpha. Virol J. 2010;7:311.

42. Bruni $R$, Marcantonio C, Tritarelli E, Tataseo P, Stellacci E, Costantino A, Villano U, Battistini A, Ciccaglione AR. An integrated approach identifies IFNregulated microRNAs and targeted mRNAs modulated by different HCV replicon clones. BMC Genomics. 2011;12:485.

43. Zhang J, Li S, Yan Q, Chen X, Yang Y, Liu X, Wan X. Interferon-beta induced microRNA-129-5p down-regulates HPV-18 E6 and E7 viral gene expression by targeting SP1 in cervical cancer cells. PLoS One. 2013;8(12):e81366.

44. Zhang J, Zhao H, Chen J, Xia B, Jin Y, Wei W, Shen J, Huang Y. Interferonbeta-induced miR-155 inhibits osteoclast differentiation by targeting SOCS1 and MITF. FEBS Lett. 2012;586(19):3255-62.

45. O'Connell RM, Taganov KD, Boldin MP, Cheng G, Baltimore D. MicroRNA-155 is induced during the macrophage inflammatory response. Proc Natl Acad Sci U S A. 2007;104(5):1604-9.

46. Otsuka M, Jing Q, Georgel P, New L, Chen J, Mols J, Kang YJ, Jiang Z, Du X, Cook R, Das SC, Pattnaik AK, Beutler B, Han J. Hypersusceptibility to vesicular stomatitis virus infection in Dicer1-deficient mice is due to impaired miR24 and miR93 expression. Immunity. 2007;27(1):123-34.

47. Bolger AM, Lohse M, Usadel B. Trimmomatic: a flexible trimmer for Illumina sequence data. Bioinformatics. 2014;30(15):2114-20.

48. Li H, Handsaker B, Wysoker A, Fennell T, Ruan J, Homer N, Marth G, Abecasis G, Durbin R. Genome project data processing S. The sequence alignment/ map format and SAMtools. Bioinformatics. 2009;25(16):2078-9.

49. Roy DG, Power AT, Bourgeois-Daigneault MC, Falls T, Ferreira L, Stern A, Tanese de Souza C, McCart JA, Stojdl DF, Lichty BD, Atkins H, Auer RC, Bell $J$ C, Le Boeuf F. Programmable insect cell carriers for systemic delivery of integrated cancer biotherapy. J Control Release. 2015;220(Pt A):210-21.

50. Pfaffl MW. A new mathematical model for relative quantification in real-time RT-PCR. Nucleic Acids Res. 2001:29(9):e45.

51. Kuleshov MV, Jones MR, Rouillard AD, Fernandez NF, Duan Q, Wang Z, Koplev S, Jenkins SL, Jagodnik KM, Lachmann A, McDermott MG, Monteiro CD, Gundersen GW, Ma'ayan A. Enrichr: a comprehensive gene set enrichment analysis web server 2016 update. Nucleic Acids Res. 2016; 44(W1):W90-7.

\section{Ready to submit your research? Choose BMC and benefit from:}

- fast, convenient online submission

- thorough peer review by experienced researchers in your field

- rapid publication on acceptance

- support for research data, including large and complex data types

- gold Open Access which fosters wider collaboration and increased citations - maximum visibility for your research: over $100 \mathrm{M}$ website views per year

At BMC, research is always in progress.

Learn more biomedcentral.com/submissions 